\title{
Numerical Radii for Tensor Products of Matrices
}

\author{
Hwa-Long $\mathrm{Gau}^{a *}$, Kuo-Zhong Wang ${ }^{b}$, Pei Yuan Wu ${ }^{b}$ \\ ${ }^{a}$ Department of Mathematics, National Central University, Chung-Li 32001, Taiwan \\ ${ }^{b}$ Department of Applied Mathematics, National Chiao Tung University, Hsinchu 30010, \\ Taiwan
}

\begin{abstract}
For $n$-by- $n$ and $m$-by- $m$ complex matrices $A$ and $B$, it is known that the inequality $w(A \otimes B) \leq\|A\| w(B)$ holds, where $w(\cdot)$ and $\|\cdot\|$ denote, respectively, the numerical radius and the operator norm of a matrix. In this paper, we consider when this becomes an equality. We show that (1) if $\|A\|=1$ and $w(A \otimes B)=w(B)$, then either $A$ has a unitary part or $A$ is completely nonunitary and the numerical range $W(B)$ of $B$ is a circular disc centered at the origin, (2) if $\|A\|=\left\|A^{k}\right\|=1$ for some $k, 1 \leq k<\infty$, then $w(A) \geq \cos (\pi /(k+2))$, and, moreover, the equality holds if and only if $A$ is unitarily similar to the direct sum of the $(k+1)$-by- $(k+1)$ Jordan block $J_{k+1}$ and a matrix $B$ with $w(B) \leq \cos (\pi /(k+2))$, and (3) if $B$ is a nonnegative matrix with its real part (permutationally) irreducible, then $w(A \otimes B)=\|A\| w(B)$ if and only if either $p_{A}=\infty$ or $n_{B} \leq p_{A}<\infty$ and $B$ is permutationally similar to a block-shift matrix

$$
\left[\begin{array}{cccc}
0 & B_{1} & & \\
& 0 & \ddots & \\
& & \ddots & B_{k} \\
& & & 0
\end{array}\right]
$$
\end{abstract}

with $k=n_{B}$, where $p_{A}=\sup \left\{\ell \geq 1:\left\|A^{\ell}\right\|=\|A\|^{\ell}\right\}$ and $n_{B}=\sup \left\{\ell \geq 1: B^{\ell} \neq 0\right\}$.

Keywords: numerical range; numerical radius; tensor product; $S_{n}$-matrix; nonnegative matrix

AMS Subject Classifications: 15A60; 15A69; 15B48

${ }^{*}$ Corresponding author. Email: hlgau@math.ncu.edu.tw 


\section{Introduction and Preliminaries}

For any $n$-by- $n$ complex matrix $A$, its numerical range $W(A)$ is, by definition, the subset $\left\{\langle A x, x\rangle: x \in \mathbb{C}^{n},\|x\|=1\right\}$ of the complex plane $\mathbb{C}$, where $\langle\cdot, \cdot\rangle$ and $\|\cdot\|$ denote the standard inner product and its associated norm in $\mathbb{C}^{n}$, respectively. The numerical radius $w(A)$ of $A$ is $\max \{|z|: z \in W(A)\}$. It is known that $W(A)$ is a nonempty compact convex subset of $\mathbb{C}$, and $w(A)$ satisfies $\|A\| / 2 \leq w(A) \leq\|A\|$, where $\|A\|$ denotes the usual operator norm of $A$. For other properties of the numerical range and numerical radius, the reader may consult [7], [9, Chapter 22] or [12, Chapter 1].

The tensor product (or Kronecker product) $A \otimes B$ of an $n$-by- $n$ matrix $A=\left[a_{i j}\right]_{i, j=1}^{n}$ and an $m$-by- $m$ matrix $B$ is the $(m n)$-by- $(m n)$ matrix

$$
\left[\begin{array}{ccc}
a_{11} B & \cdots & a_{1 n} B \\
\vdots & & \vdots \\
a_{n 1} B & \cdots & a_{n n} B
\end{array}\right] .
$$

It is known that $A \otimes B$ and $B \otimes A$ are unitarily similar and $\|A \otimes B\|=\|A\| \cdot\|B\|$. Other properties of the tensor product can be found in [12, Chapter 4].

The main concern of this paper is the relations between the numerical radius of $A \otimes$ $B$ and those of $A$ and $B$. For one direction, we have $w(A \otimes B) \leq \min \{\|A\| w(B),\|B\| w(A)\}$. This can be proven by using the unitary dilation of contractions, as to be done below. On the other hand, we also have $w(A \otimes B) \geq w(A) w(B)$. We are interested in when these become equalities. In the present paper, we obtain various conditions, necessary or sufficient, for $w(A \otimes B)=\|A\| w(B)$ to hold. The discussions on the equality $w(A \otimes B)=w(A) w(B)$ will be the subject of a subsequent paper of ours.

For the ease of exposition, we introduce two indices for an $n$-by- $n$ matrix $A$ : the power norm index $p_{A}$ and nilpotency index $n_{A}$ of $A$. They are defined, respectively, by

$$
p_{A}=\sup \left\{k \geq 1:\left\|A^{k}\right\|=\|A\|^{k}\right\}
$$


and

$$
n_{A}= \begin{cases}\sup \left\{k \geq 1: A^{k} \neq 0_{n}\right\} & \text { if } A \neq 0_{n}, \\ 0 & \text { if } A=0_{n},\end{cases}
$$

where $0_{n}$ denotes the $n$-by- $n$ zero matrix.

We start in Section 2 by proving that if $\|A\|=1$ and $w(A \otimes B)=w(B)$, then either $A$ has a unitary part or $A$ is completely nonunitary and $W(B)$ is a circular disc centered at the origin (Theorem 2.2). The proof depends on the dilation of $A$

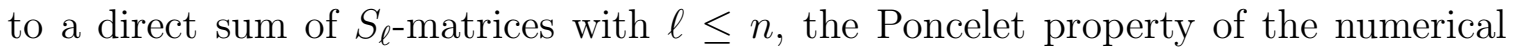
ranges of matrices of the latter class, and Anderson's theorem on the circular disc numerical range. As a by-product, we obtain a lower bound for $w(A)$ when $A$ satisfies $\|A\|=\left\|A^{k}\right\|=1$ for some $k, 1 \leq k<n: w(A) \geq \cos (\pi /(k+2))$, and determine exactly when this bound is attained: this is the case if and only if $A$ is unitarily similar to $J_{k+1} \oplus B$, where $J_{k+1}$ is the $(k+1)$-by- $(k+1)$ Jordan block

$$
\left[\begin{array}{llll}
0 & 1 & & \\
& 0 & \ddots & \\
& & \ddots & 1 \\
& & & 0
\end{array}\right]
$$

and $B$ is a finite matrix with $w(B) \leq \cos (\pi /(k+2)$ ) (Theorem 2.10). This generalizes the classical result of Willams and Crimmins [17] for $k=1$. We conclude Section 2 with a result on nilpotent contractions, namely, we prove that if $A$ is an $n$-by- $n$ matrix with $\|A\|=1$, then a necessary and sufficient condition for $p_{A}=n_{A}<\infty$ to hold is that $A$ be unitarily similar to a direct sum $J_{k+1} \oplus B$, where $k=p_{A}$ and $B^{k+1}=0$ (Theorem 2.13).

Finally, in Section 3, we consider $B$ to be a nonnegative matrix with $\operatorname{Re} B(=$ $\left(B+B^{*}\right) / 2$ ) (permutationally) irreducible. We obtain in Theorem 3.1 a complete characterization for $w(A \otimes B)=\|A\| w(B)$, namely, this is the case if and only if either $p_{A}=\infty$ or $n_{B} \leq p_{A}<\infty$ and $B$ is permutationally similar to a block-shift 
matrix of the form

$$
\left[\begin{array}{cccc}
0 & B_{1} & & \\
& 0 & \ddots & \\
& & \ddots & B_{k} \\
& & & 0
\end{array}\right]
$$

with $k=n_{B}$.

As was mentioned before, the inequality $w(A \otimes B) \leq\|A\| w(B)$ for $n$-by- $n$ and $m$-by- $m$ matrices $A$ and $B$ is known. It is a consequence of [10, Theorem 3.4] because $A \otimes B$ is the product of $A \otimes I_{m}$ and $I_{n} \otimes B$, and the latter two matrices doubly commute, that is, $A \otimes I_{m}$ commutes with both $I_{n} \otimes B$ and its adjoint $I_{n} \otimes B^{*}$. Here we give a simple proof based on the unitary dilation of contractions.

Proposition 1.1. If $A$ and $B$ are $n$-by-n and $m$-by-m matrices, respectively, then $w(A \otimes B) \leq \min \{\|A\| w(B),\|B\| w(A)\}$.

Proof. We need only prove that $w(A \otimes B) \leq\|A\| w(B)$, and may assume that $\|A\|=1$. Then the $(2 n)$-by- $(2 n)$ matrix

$$
U=\left[\begin{array}{cc}
A & \left(I_{n}-A A^{*}\right)^{1 / 2} \\
\left(I_{n}-A^{*} A\right)^{1 / 2} & -A^{*}
\end{array}\right]
$$

is unitary. Let $U$ be unitarily similar to the diagonal matrix $\operatorname{diag}\left(u_{1}, \ldots, u_{2 n}\right)$, where $\left|u_{j}\right|=1$ for all $j$. Then

$$
w(A \otimes B) \leq w(U \otimes B)=w\left(\sum_{j=1}^{2 n} \oplus u_{j} B\right)=\max _{j} w\left(u_{j} B\right)=w(B)=\|A\| w(B) .
$$

We conclude this section with some basic properties of the indices $p_{A}$ and $n_{A}$ of a matrix $A$.

Proposition 1.2. Let $A$ be an $n-b y-n$ matrix. Then

(a) $1 \leq p_{A} \leq n-1$ or $p_{A}=\infty$, 
(b) $p_{A}=n-1$ if and only if $A$ is a nonzero multiple of a $S_{n}$-matrix, and

(c) the following conditions are equivalent:

(1) $p_{A}=\infty$,

(2) $\|A\|=\rho(A)$,

(3) $\|A\|=w(A)$,

and if $\|A\|=1$, then the above are also equivalent to

(4) A has a unitary part.

Here $\rho(A)$ denotes the spectral radius of $A$, that is, $\rho(A)$ is the maximum modulus of eigenvalues of $A$.

Recall that an $n$-by- $n$ matrix $A$ is of class $S_{n}$ if it is a contraction $(\|A\| \leq 1)$, its eigenvalues are all in $\mathbb{D} \equiv\{z \in \mathbb{C}:|z|<1\}$, and $\operatorname{rank}\left(I_{n}-A^{*} A\right)=1$. Any contraction $A$ is unitarily similar to the direct sum of a unitary matrix $U$, called the unitary part of $A$, and a completely nonunitary contraction $A^{\prime}$, called the c.n.u. part of $A$. The latter means that $A^{\prime}$ is not unitarily similar to any direct sum with a unitary summand.

Proof of Proposition 1.2. (a) was obtained by Pták in 1960 (cf. [15, Theorem 2.1]) and (b) was proven in [4, Theorem 3.1]. As for (c), the implication (1) $\Rightarrow(2)$ is by [9, Problem 88], (2) $\Rightarrow(3)$ by the known inequalities $\rho(A) \leq w(A) \leq\|A\|,(3) \Rightarrow(2)$ by [9, Problem 218 (b)], and (2) $\Rightarrow(1)$ by the inequalities $\rho(A) \leq\left\|A^{k}\right\|^{1 / k} \leq\|A\|$ for all $k \geq 1$. If $\|A\|=\rho(A)=1$, then, letting $\lambda$ be an eigenvalue of $A$ with $|\lambda|=1$, we have the unitary similarity of $A$ and a matrix of the form $\left[\begin{array}{ll}\lambda & B \\ 0 & C\end{array}\right]$. Since $\|A\|=|\lambda|=1$ implies that $B=0, A$ is unitarily similar to $[\lambda] \oplus C$ and thus has a unitary part. This proves $(2) \Rightarrow(4)$. That $(4) \Rightarrow(2)$ is trivial.

Proposition 1.3. Let $A$ be an n-by-n matrix. Then 
(a) $0 \leq n_{A} \leq n-1$ or $n_{A}=\infty$,

(b) $n_{A}=n-1$ if and only if $A$ is similar to the $n$-by-n Jordan block $J_{n}$,

(c) $n_{A}=\infty$ if and only if $A$ is not nilpotent, and

(d) $p_{A} \leq n_{A}$ for $A \neq 0_{n}$.

We omit its easy proofs.

In the following, we use $\sigma(A)$ to denote the spectrum of $A$, that is, $\sigma(A)$ is the set of eigenvalues of $A$. An $n$-by- $n$ matrix $A$ is a dilation of an $m$-by- $m$ matrix $B$ (or $B$ is a compression of $A$ ) if there is an $n$-by- $m$ matrix $V$ such that $B=V^{*} A V$ and $V^{*} V=I_{m}$. This is equivalent to $A$ being unitarily similar to a matrix of the form $\left[\begin{array}{ll}B & * \\ * & *\end{array}\right]$

\section{Contractions}

We start with a simple condition which yields the equality $w(A \otimes B)=\|A\| w(B)$.

Lemma 2.1. If $A$ is an n-by-n matrix with $p_{A}=\infty$, then $w(A \otimes B)=\|A\| w(B)$ for any $m$-by-m matrix $B$. In particular, this is the case for $A$ a contraction with a unitary part.

Proof. Since $p_{A}=\infty$ implies, by Proposition 1.2 (c), that $\|A\|=w(A)$. If $\lambda$ is a number in $W(A)$ with $|\lambda|=w(A)$, then $|\lambda|=\|A\|$. Since $A$ is unitarily similar to a matrix of the form $\left[\begin{array}{ll}\lambda & * \\ * & *\end{array}\right]$, we have the unitary similarity of $A \otimes B$ and $\left[\begin{array}{cc}\lambda B & * \\ * & *\end{array}\right]$. It follows that $\|A\| w(B)=w(\lambda B) \leq w(A \otimes B)$. On the other hand, we also have $w(A \otimes B) \leq\|A\| w(B)$ by Proposition 1.1. Thus $w(A \otimes B)=\|A\| w(B)$ holds.

The next theorem is one of the main results of this section. It gives a necessary condition for the equality $w(A \otimes B)=\|A\| w(B)$. 
Theorem 2.2. Let $A$ and $B$ be $n$-by-n and $m$-by-m matrices, respectively. If $\|A\|=1$ and $w(A \otimes B)=w(B)$, then either $A$ has a unitary part or $A$ is c.n.u. and $W(B)$ is a circular disc centered at the origin.

We first prove this for the case when $A$ is an $S_{n}$-matrix. The numerical ranges of such matrices are known to have the Poncelet property, namely, if $A$ is of class $S_{n}$, then, for any point $\lambda$ on the unit circle $\partial \mathbb{D}$, there is a unique (up to unitary similarity) $(n+1)$-by- $(n+1)$ unitary dilation $U$ of $A$ such that $\lambda$ is an eigenvalue of $U$ and each edge of the $(n+1)$-gon $\partial W(U)$ intersects $W(A)$ at exactly one point (cf. 2, Theorem 2.1 and Lemma 2.2]).

Lemma 2.3. Let $A$ be an $S_{n}$-matrix and $B$ an $m$-by-m matrix. If $w(A \otimes B)=w(B)$, then $W(B)$ is a circular disc centered at the origin.

Proof. Let $U_{1}, \ldots, U_{m+1}$ be $(n+1)$-by- $(n+1)$ unitary dilations of $A$ with $\sigma\left(U_{i}\right) \cap$ $\sigma\left(U_{j}\right)=\emptyset$ for all $i$ and $j, 1 \leq i \neq j \leq m+1$. We may assume that $U_{j}=$ $\operatorname{diag}\left(\lambda_{1 j}, \ldots, \lambda_{n+1, j}\right)$ for each $j$, where $\left|\lambda_{i j}\right|=1$ for all $i$ and $j$. Let $V_{j}$ be an $(n+1)$ by-n matrix such that $A=V_{j}^{*} U_{j} V_{j}$ and $V_{j}^{*} V_{j}=I_{n}$ for each $j$. Since $\|A\|=1$ and

$$
w(A \otimes \lambda B)=w(A \otimes B)=w(B)=w(\lambda B)
$$

for any $\lambda,|\lambda|=1$, we may further assume that $w(B)$ is in $W(A \otimes B)$. Let $x$ be a unit vector in $\mathbb{C}^{n} \otimes \mathbb{C}^{m}$ such that $\langle(A \otimes B) x, x\rangle=w(B)$. We decompose $\left(V_{j} \otimes I_{m}\right) x$ as $y_{1 j} \oplus \cdots \oplus y_{n+1, j}$ with $y_{i j}, 1 \leq i \leq n+1$, in $\mathbb{C}^{m}$ for each $j$. Then

$$
\begin{aligned}
w(B) & =\langle(A \otimes B) x, x\rangle \\
& =\left\langle\left(U_{j} \otimes B\right)\left(V_{j} \otimes I_{m}\right) x,\left(V_{j} \otimes I_{m}\right) x\right\rangle \\
& =\left\langle\left(\lambda_{1 j} B \oplus \cdots \oplus \lambda_{n+1, j} B\right)\left(y_{1 j} \oplus \cdots \oplus y_{n+1, j}\right), y_{1 j} \oplus \cdots \oplus y_{n+1, j}\right\rangle \\
& =\sum_{i=1}^{n+1}\left\langle\lambda_{i j} B y_{i j}, y_{i j}\right\rangle \\
& \leq \sum_{i=1}^{n+1}\left|\left\langle B y_{i j}, y_{i j}\right\rangle\right| .
\end{aligned}
$$


Letting $\eta_{i j}=\left\langle B\left(y_{i j} /\left\|y_{i j}\right\|\right), y_{i j} /\left\|y_{i j}\right\|\right\rangle$ for each $y_{i j} \neq 0$, we obtain

$$
w(B)=\sum_{y_{i j} \neq 0} \lambda_{i j}\left\|y_{i j}\right\|^{2} \eta_{i j} \leq \sum_{y_{i j} \neq 0}\left\|y_{i j}\right\|^{2}\left|\eta_{i j}\right| \leq \sum_{y_{i j} \neq 0}\left\|y_{i j}\right\|^{2} w(B)=w(B)
$$

since

$$
\sum_{i}\left\|y_{i j}\right\|^{2}=\left\|\left(V_{j} \otimes I_{m}\right) x\right\|^{2}=\|x\|^{2}=1 .
$$

Thus we have equalities throughout the above sequence, which yields that $w(B)=$ $\lambda_{i j} \eta_{i j}$ for $y_{i j} \neq 0$. Since $\sum_{i}\left\|y_{i j}\right\|^{2}=1$, this must hold for at least one $i$, say, $i_{j}$. Hence $\bar{\lambda}_{i_{j} j} w(B)=\eta_{i_{j} j}$ is in $\partial W(B)$ for each $j$. Note that such $\bar{\lambda}_{i_{j} j} w(B)$ 's, $1 \leq j \leq m+1$, are distinct from each other by our assumption on the disjointness of the spectra of the $U_{j}$ 's. This shows that the boundary of $W(B)$ and the circle $|z|=w(B)$ intersect at at least $m+1$ points. Since $W(B)$ is contained in $\{z \in \mathbb{C}:|z| \leq w(B)\}$, we apply Anderson's theorem (cf. [3, Theorem] or [20]) to infer that $W(B)=\{z \in \mathbb{C}:|z| \leq$ $w(B)\}$.

Proof of Theorem 2.2. We assume that $A$ is c.n.u. Then $A$ can be dilated to the direct $\operatorname{sum} A^{\prime} \oplus \cdots \oplus A^{\prime}$ of $\operatorname{rank}\left(I_{n}-A^{*} A\right)$ many copies of some $S_{\ell^{-}}$matrix $A^{\prime}$ with $\ell \leq n$ (cf. [18, Theorem 1.4] or [21, Lemma $3(\mathrm{a})])$. Hence $A \otimes B$ dilates to $\left(A^{\prime} \oplus \cdots \oplus A^{\prime}\right) \otimes B=$ $\left(A^{\prime} \otimes B\right) \oplus \cdots \oplus\left(A^{\prime} \otimes B\right)$. We have

$w(B)=w(A \otimes B) \leq w\left(\left(A^{\prime} \otimes B\right) \oplus \cdots \oplus\left(A^{\prime} \otimes B\right)\right)=w\left(A^{\prime} \otimes B\right) \leq\left\|A^{\prime}\right\| w(B)=w(B)$.

Thus $w\left(A^{\prime} \otimes B\right)=w(B)$. It follows from Lemma 2.3 that $W(B)$ is a circular disc centered at the origin.

An easy consequence of Theorem 2.2 is that the converse of Lemma 2.1 is also true.

Corollary 2.4. For an $n$-by-n matrix $A$, the equality $w(A \otimes B)=\|A\| w(B)$ holds for all matrices $B$ if and only if $p_{A}=\infty$. 
Proof. For the necessity, assume that $\|A\|=1$ and let $B$ be any matrix with its numerical range not a circular disc centered at the origin. Theorem 2.2 yields that $A$ has a unitary part. Then $p_{A}=\infty$ follows immediately.

In Theorem 2.2, if $B$ is the Jordan block $J_{m}$, then we have the following characterizations for $w(A \otimes B)=\|A\| w(B)$.

Theorem 2.5. Let $A$ be an $n$-by-n matrix with $\|A\|=1$. Then the following conditions are equivalent:

(a) $W\left(A \otimes J_{m}\right)=W\left(J_{m}\right)$,

(b) $w\left(A \otimes J_{m}\right)=w\left(J_{m}\right)$,

(c) $A \otimes J_{m}$ is unitarily similar to $J_{m} \oplus B$ for some matrix $B$ with $w(B) \leq w\left(J_{m}\right)$, and

(d) $\left\|A^{m-1}\right\|=1$.

If, in addition, $n=m$, then the above conditions are also equivalent to

(e) either $A$ has a unitary part or $A$ is of class $S_{n}$, and

(f) $p_{A}=\infty$ or $n-1$.

Note that $W\left(J_{m}\right)=\{z \in \mathbb{C}:|z| \leq \cos (\pi /(m+1))\}$ (cf. [8, Proposition 1]).

Proof of Theorem 2.5. The implication (a) $\Rightarrow(\mathrm{b})$ is trivial. To prove (b) $\Rightarrow(\mathrm{c})$, note that $\left(A \otimes J_{m}\right)^{m}=A^{m} \otimes J_{m}^{m}=0_{n m}$ and $\left\|A \otimes J_{m}\right\|=\|A\|\left\|J_{m}\right\|=1$. If $x$ is a unit vector in $\mathbb{C}^{n} \otimes \mathbb{C}^{m}$ such that $\left|\left\langle\left(A \otimes J_{m}\right) x, x\right\rangle\right|=w\left(A \otimes J_{m}\right)$, then $w\left(A \otimes J_{m}\right)=w\left(J_{m}\right)=$ $\cos (\pi /(m+1))$ implies that the subspace $K$ of $\mathbb{C}^{n} \otimes \mathbb{C}^{m}$ generated by the vectors $x,\left(A \otimes J_{m}\right) x, \ldots,\left(A \otimes J_{m}\right)^{m-1} x$ is reducing for $A \otimes J_{m}$, and the restriction of $A \otimes J_{m}$ to $K$ is unitarily similar to $J_{m}$ (cf. [8, Theorem 1 (2)]). Hence $A \otimes J_{m}$ is unitarily 
similar to $J_{m} \oplus B$, where $B$ is the restriction of $A \otimes J_{m}$ to $K^{\perp}$. We obviously have $w(B) \leq w\left(A \otimes J_{m}\right)=w\left(J_{m}\right)$.

For $(\mathrm{c}) \Rightarrow(\mathrm{d})$, note that $A^{m-1} \otimes J_{m}^{m-1}$ is unitarily similar to $J_{m}^{m-1} \oplus B^{m-1}$ under (c). Hence

$$
\left\|A^{m-1}\right\|=\left\|A^{m-1} \otimes J_{m}^{m-1}\right\|=\left\|J_{m}^{m-1} \oplus B^{m-1}\right\|=\max \left\{\left\|J_{m}^{m-1}\right\|,\left\|B^{m-1}\right\|\right\}=1 .
$$

To prove $(\mathrm{d}) \Rightarrow(\mathrm{c})$, let $x$ be a unit vector in $\mathbb{C}^{n}$ such that $\left\|A^{m-1} x\right\|=1$. Then $\left\|A^{m-j} x\right\|=1$ for all $j, 1 \leq j \leq m$. Let $\left\{e_{1}, \ldots, e_{m}\right\}$ be the standard basis for $\mathbb{C}^{m}$, let $x_{j}=A^{m-j} x \otimes e_{j}, 1 \leq j \leq m$, and let $K$ be the subspace of $\mathbb{C}^{n} \otimes \mathbb{C}^{m}$ generated by $x_{1}, \ldots, x_{m}$. Then $\left(A \otimes J_{m}\right) x_{1}=0$ and $\left(A \otimes J_{m}\right) x_{j}=x_{j-1}$ for $2 \leq j \leq m$. Since $\left\{x_{1}, \ldots, x_{m}\right\}$ is an orthonormal basis of $K$, this shows that $\left(A \otimes J_{m}\right) K \subseteq K$ and the restriction of $A \otimes J_{m}$ to $K$ is unitarily similar to $J_{m}$. On the other hand, it follows from $\left\|A \otimes J_{m}\right\|=\|A\|\left\|J_{m}\right\|=1$ and

$$
\left(A \otimes J_{m}\right)^{*} x_{m}=\left(A^{*} \otimes J_{m}^{*}\right)\left(x \otimes e_{m}\right)=\left(A^{*} x\right) \otimes\left(J_{m}^{*} e_{m}\right)=\left(A^{*} x\right) \otimes 0=0
$$

that $K$ is reducing for $A \otimes J_{m}$, and hence $A \otimes J_{m}$ is unitarily similar to $J_{m} \oplus B$, where $B$ is the restriction of $A \otimes J_{m}$ to $K^{\perp}$. Obviously, we have

$$
w(B) \leq w\left(A \otimes J_{m}\right) \leq\|A\| w\left(J_{m}\right)=w\left(J_{m}\right)
$$

To prove $(\mathrm{c}) \Rightarrow(\mathrm{a})$, note that the unitary similarity of $J_{m}$ and $e^{i \theta} J_{m}$ for all real $\theta$ implies the same for $A \otimes J_{m}$ and $e^{i \theta}\left(A \otimes J_{m}\right)$. Thus $W\left(A \otimes J_{m}\right)$ is a circular disc centered at the origin. (c) implies that $w\left(A \otimes J_{m}\right)=w\left(J_{m}\right)$, which means that the radii of the two circular discs $W\left(A \otimes J_{m}\right)$ and $W\left(J_{m}\right)$ are equal. Therefore, $W\left(A \otimes J_{m}\right)=W\left(J_{m}\right)$ holds.

Now assume that $n=m$ and that $\left\|A^{n-1}\right\|=1$. If $\left\|A^{n}\right\|=1$, then $p_{A}=\infty$ and hence $A$ has a unitary part by Proposition 1.2 (a) and (c). On the other hand, if $\left\|A^{n}\right\|<1$, then $A$ is of class $S_{n}$ by [4, Theorem 3.1]. This shows that $(\mathrm{d}) \Rightarrow(\mathrm{e})$. Next, if (e) is true, then $p_{A}=\infty$ or $n-1$ depending on whether $A$ has a unitary part 
or $A$ is of class $S_{n}$ (cf. [4, Theorem 3.1] for the latter). This proves (f). Finally, if $p_{A}=\infty$, then $\left\|A^{k}\right\|=1$ for all $k \geq 1$, and, in particular, $\left\|A^{n-1}\right\|=1$. On the other hand, if $p_{A}=n-1$, then $\left\|A^{n-1}\right\|=\|A\|^{n-1}=1$. This proves (f) $\Rightarrow(\mathrm{d})$.

The next proposition gives a characterization of $w(A \otimes B)=\|A\| w(B)$ when $B$ is of class $S_{m}$.

Proposition 2.6. Let $A$ be an $n$-by-n matrix with $\|A\|=1$, and $B$ be an $S_{m}$-matrix. Then $w(A \otimes B)=w(B)$ if and only if either $A$ has a unitary part or $A$ is c.n.u., $\left\|A^{m-1}\right\|=1$ and $B$ is unitarily similar to $J_{m}$.

Its proof depends on a special property of $S_{n}$-matrices. The following lemma is from [19, Lemma 5]. Here we give a shorter geometric proof.

Lemma 2.7. Let $A$ be an $S_{n}$-matrix. Then $W(A)$ is a circular disc centered at the origin if and only if $A$ is unitarily similar to $J_{n}$.

Proof. If $W(A)$ is as asserted, then the Poncelet property of $W(A)$ says that it is circumscribed by $(n+1)$-gons with vertices on the unit circle. As the circular disc $\{z \in \mathbb{C}:|z| \leq \cos (\pi /(n+1))\}\left(=W\left(J_{n}\right)\right)$ is circumscribed by any regular $(n+1)$-gon on the unit circle, if the radius of $W(A)$ is not equal to $\cos (\pi /(n+1))$, then we infer from a geometrical consideration that $W(A)$ cannot have the Poncelet property. Thus $W(A)$ must equal $W\left(J_{n}\right)$. The unitary similarity of $A$ and $J_{n}$ then follows from [2, Theorem 3.2]. The converse is trivial.

Proof of Proposition 2.6. If $w(A \otimes B)=w(B)$, then, by Theorem 2.2, either $A$ has a unitary part or $A$ is c.n.u. and $W(B)$ is a circular disc centered at the origin. In the latter case, Lemma 2.7 yields the unitary similarity of $B$ and $J_{m}$, and then Theorem 2.5 gives $\left\|A^{m-1}\right\|=1$. The converse also follows from Theorem 2.5. 
Note that, under the conditions of Proposition 2.6, if $A$ is c.n.u., then we automatically have $m \leq n$. This is because if, otherwise, $m>n$, then $\left\|A^{m-1}\right\|=1$ yields, by Proposition 1.2 (a) and (c), that $A$ has a unitary part.

A specific example of the results obtained so far is in the next proposition.

Proposition 2.8. Let $n$ and $m$ be positive integers. Then $W\left(J_{n} \otimes J_{m}\right)=W\left(J_{\ell}\right)$, where $\ell=\min \{n, m\}$, and thus $w\left(J_{n} \otimes J_{m}\right)=\min \left\{w\left(J_{n}\right), w\left(J_{m}\right)\right\}$.

Proof. Assume that $m \leq n$. Since the principal submatrix of $J_{n} \otimes J_{m}$ formed by its rows and columns numbered $1, m+2,2 m+3, \ldots$, and $(m-1) m+m$ is $J_{m}$, we have that $J_{n} \otimes J_{m}$ is a dilation of $J_{m}$. Thus $w\left(J_{m}\right) \leq w\left(J_{n} \otimes J_{m}\right)$. The reversed inequality $w\left(J_{n} \otimes J_{m}\right) \leq\left\|J_{n}\right\| w\left(J_{m}\right)=w\left(J_{m}\right)$ is by Proposition 1.1. Therefore, $w\left(J_{n} \otimes J_{m}\right)=w\left(J_{m}\right)$ holds. As was seen in the proof of (c) $\Rightarrow$ (a) in Theorem 2.5, $W\left(J_{n} \otimes J_{m}\right)$ is a circular disc centered at the origin. Thus the equality of $w\left(J_{n} \otimes J_{m}\right)$ and $w\left(J_{m}\right)$ implies that of $W\left(J_{n} \otimes J_{m}\right)$ and $W\left(J_{m}\right)$.

Besides $S_{n}$-matrices, another generalization of the Jordan blocks is the companion matrices. Recall that a companion matrix is one of the form

$$
\left[\begin{array}{ccccccc}
0 & 1 & & & & \\
& 0 & 1 & & & \\
& & \cdot & \cdot & & \\
& & & \cdot & & \\
& & & \cdot & \cdot & \\
& & & & 0 & 1 \\
-a_{n} & -a_{n-1} & \cdot & \cdot & \cdot & -a_{2} & -a_{1}
\end{array}\right],
$$

whose characteristic and minimal polynomials are both equal to $z^{n}+\sum_{j=1}^{n} a_{j} z^{n-j}$. The numerical ranges of such matrices have been studied in [5, 6, 1]. 
Proposition 2.9. Let $A$ be an n-by-n $(n \geq 2)$ companion matrix. Then the following conditions are equivalent:

(a) $w(A \otimes A)=\|A\| w(A)$,

(b) $A$ is unitary, $A=J_{n}$, or $A$ is unitarily similar to a direct sum $\left[a \omega_{n}^{j}\right] \oplus B$, where $|a|>1, \omega_{n}=e^{i(2 \pi / n)}, 0 \leq j \leq n-1$, and $B$ is an $S_{n-1}$-matrix with eigenvalues $(1 / \bar{a}) \omega_{n}^{k}, 0 \leq k \leq n-1$ and $k \neq j$, and

(c) $p_{A}=n_{A}=\infty$ or $n-1$.

Proof. To prove (a) $\Rightarrow(\mathrm{b})$, let $A^{\prime}=A /\|A\|$. Then (a) gives $w\left(A^{\prime} \otimes A^{\prime}\right)=w\left(A^{\prime}\right)$. By Theorem 2.2, either $A^{\prime}$ has a unitary part or it is c.n.u. with numerical range a circular disc centered at the origin. In the former case, either $A$ is normal or is unitarily similar to a matrix of the form $\left[a \omega_{n}^{j}\right] \oplus B$, where $|a|=\|A\| \geq 1$ and $B$ is of size $n-1$ with eigenvalues $(1 / \bar{a}) \omega_{n}^{k}, 0 \leq k \leq n-1$ and $k \neq j$ (cf. [5, Theorem 1.1 and Corollary 1.3]). If $A$ is normal or $|a|=1$, then $A$ is unitary by [5, Corollary 1.2]. Hence we may assume that $|a|>1$. Thus the eigenvalues of $B$ are all contained in $\mathbb{D}$. Moreover, by [1, Theorem 2.1], we have $\operatorname{rank}\left(I_{n-1}-B^{*} B\right)=1$. These two together imply, by way of the singular value decomposition of $B$, that $\|B\|=1$. Hence $B$ is of class $S_{n-1}$. On the other hand, if it is the latter case, then $W(A)$ is also a circular disc centered at the origin. Therefore, $A=J_{n}$ by [5, Theorem 2.9]. This proves (b).

For (b) $\Rightarrow\left(\mathrm{c}\right.$ ), if $A$ is unitary (resp., $A=J_{n}$ ), then, obviously, $p_{A}=n_{A}=\infty$ (resp., $p_{A}=n_{A}=n-1$ ). On the other hand, if $A$ is unitarily similar to the asserted $\left[a \omega_{n}^{j}\right] \oplus B$, then $\|A\|=\max \{|a|,\|B\|\}=|a|=\rho(A)$. Thus $p_{A}=n_{A}=\infty$ by Proposition 1.2 (c) and 1.3.

Finally, for (c) $\Rightarrow$ (a), if $p_{A}=n_{A}=\infty$, then (a) is a consequence of Lemma 2.1. On the other hand, if $p_{A}=n_{A}=n-1$, then $A^{n}=0_{n}$. This implies that $A=J_{n}$ and thus (a) holds by Proposition 2.8 . 
The next theorem is a consequence of Theorem 2.5. It gives a lower bound, in terms of $p_{A}$, for $w(A)$ when $A$ is an $n$-by- $n$ matrix with $\|A\|=1$.

Theorem 2.10. If $A$ is an $n$-by-n matrix with $\|A\|=\left\|A^{k}\right\|=1$ for some $k \geq 1$, then $w(A) \geq \cos (\pi /(k+2))$. Moreover, in this case, the following conditions are equivalent:

(a) $w(A)=\cos (\pi /(k+2))$,

(b) $A$ is unitarily similar to $J_{k+1} \oplus B$, where $B$ is a finite matrix with $w(B) \leq$ $\cos (\pi /(k+2))$, and

(c) $W(A)=\{z \in \mathbb{C}:|z| \leq \cos (\pi /(k+2))\}$.

For the proof of $(\mathrm{a}) \Rightarrow(\mathrm{b})$, we need the following lemma.

Lemma 2.11. Let

$$
A=\left[\begin{array}{ccccc}
0 & a_{1} & & & \\
& 0 & \ddots & & \\
& & \ddots & a_{n-2} & \\
& & & 0 & a_{n-1} \\
& & & & a
\end{array}\right] \text { and } B=\left[\begin{array}{cccc}
0 & a_{1} & & \\
& 0 & \ddots & \\
& & \ddots & a_{n-2} \\
& & & 0
\end{array}\right]
$$

be $n$-by- $n$ and $(n-1)$-by- $(n-1)$ matrices, respectively, where $n \geq 2$ and $a_{j}$ is nonzero for all $j$. Then $w(A)>w(B)$.

Proof. We prove this by induction on $n$. If $n=2$, then $A=\left[\begin{array}{cc}0 & a_{1} \\ 0 & a\end{array}\right]$ and $B=[0]$, in which case we obviously have $w(A)>0=w(B)$. Assume now that the assertion is true for the matrix $A$ of size at most $n-1(n \geq 3)$, and let $A$ and $B$ be of the above form. By considering $e^{i \theta} A$ for a suitable real $\theta$ instead of $A$, we may assume 
that $w(A)$ equals the largest eigenvalue of $\operatorname{Re} A$. Let

$$
C=\left[\begin{array}{cccc}
0 & a_{1} & & \\
& 0 & \ddots & \\
& & \ddots & a_{n-3} \\
& & & 0
\end{array}\right],
$$

and let $p(z), q(z)$ and $r(z)$ be the characteristic polynomials of $\operatorname{Re} A, \operatorname{Re} B$ and $\operatorname{Re} C$, respectively. We expand the determinant of

$$
\left[\begin{array}{ccccc}
z & -a_{1} / 2 & & & \\
-\bar{a}_{1} / 2 & z & \ddots & & \\
& \ddots & \ddots & \ddots & \\
& & \ddots & z & -a_{n-1} / 2 \\
& & & -\bar{a}_{n-1} / 2 & z-\operatorname{Re} a
\end{array}\right]
$$

by minors on its last row to obtain $p(z)=(z-\operatorname{Re} a) q(z)-\left(\left|a_{n-1}\right|^{2} / 4\right) r(z)$. Let $\alpha, \beta$ and $\gamma$ be the largest eigenvalues of $\operatorname{Re} A, \operatorname{Re} B$ and $\operatorname{Re} C$, respectively. Then $\alpha=w(A), \beta=w(B)$ and $\gamma=w(C)$. Since $\operatorname{Re} B$ (resp., $\operatorname{Re} C$ ) is a principal submatrix of $\operatorname{Re} A$ (resp., $\operatorname{Re} B$ ), we have $\beta \leq \alpha$ (resp., $\gamma \leq \beta$ ). Assume that $\alpha=\beta$. Then the above equation yields

$$
0=p(\alpha)=(\alpha-\operatorname{Re} a) q(\beta)-\frac{1}{4}\left|a_{n-1}\right|^{2} \gamma(\beta)=-\frac{1}{4}\left|a_{n-1}\right|^{2} \gamma(\beta) .
$$

Since $a_{n-1} \neq 0$ and $\beta$ is larger than or equal to all eigenvalues of $\operatorname{Re} C$, we infer from $\gamma(\beta)=0$ that $\beta=\gamma$ or $w(B)=w(C)$. This contradicts our induction hypothesis for $B$ and $C$. Hence we must have $\alpha>\beta$ or $w(A)>w(B)$.

Proof of Theorem 2.10. By Theorem 2.5, the assumption $\|A\|=\left\|A^{k}\right\|=1$ implies that $w\left(A \otimes J_{k+1}\right)=w\left(J_{k+1}\right)$. Hence

$$
w(A)=\left\|J_{k+1}\right\| w(A) \geq w\left(A \otimes J_{k+1}\right)=w\left(J_{k+1}\right)=\cos \frac{\pi}{k+2}
$$

as asserted. 
We now prove the equivalence of (a), (b) and (c). The implications (b) $\Rightarrow$ (c) and $(\mathrm{c}) \Rightarrow(\mathrm{a})$ are trivial. To prove $(\mathrm{a}) \Rightarrow(\mathrm{b})$, let $x$ be a unit vector in $\mathbb{C}^{n}$ such that $\left\|A^{k} x\right\|=1$. Then $\left\|A^{j} x\right\|=1$ for all $j, 0 \leq j \leq k$. We now check that $A^{k+1} x=0$. Assuming otherwise that $\left\|A^{k+1} x\right\|>0$, let $u_{t}=\left[u_{t 1} \ldots u_{t, k+2}\right]^{T}$ in $\mathbb{C}^{k+2} \otimes \mathbb{C}^{n}$, where

$$
u_{t j}= \begin{cases}\frac{\sqrt{1-t^{2}}}{\left\|A^{k+1} x\right\|} A^{k+1} x & \text { if } j=1, \\ t \sqrt{\frac{2}{k+2}} \sin \frac{(j-1) \pi}{k+2} A^{k-j+2} x & \text { if } j=2, \ldots, k+2\end{cases}
$$

for any $t, 0<t<1$. Note that

$$
v \equiv \sqrt{\frac{2}{k+2}}\left[\begin{array}{llll}
\sin \frac{\pi}{k+2} & \sin \frac{2 \pi}{k+2} & \ldots & \sin \frac{(k+1) \pi}{k+2}
\end{array}\right]^{T}
$$

is a unit vector in $\mathbb{C}^{k+1}$ with $\left\langle J_{k+1} v, v\right\rangle=\cos (\pi /(k+2))$ (cf. [8, Proposition 1 (3)]). Hence $\left\|u_{t}\right\|=\left(\left(1-t^{2}\right)+t^{2}\|v\|^{2}\right)^{1 / 2}=1$, and

$$
\begin{aligned}
\left\langle\left(J_{k+2} \otimes A\right) u_{t}, u_{t}\right\rangle= & t \sqrt{1-t^{2}} \sqrt{\frac{2}{k+2}} \sin \frac{\pi}{k+2}\left\|A^{k+1} x\right\| \\
& +t^{2} \frac{2}{k+2} \sum_{j=1}^{k} \sin \frac{j \pi}{k+2} \sin \frac{(j+1) \pi}{k+2}\left\|A^{k-j+1} x\right\|^{2} \\
= & t \sqrt{1-t^{2}} \sqrt{\frac{2}{k+2}} \sin \frac{\pi}{k+2}\left\|A^{k+1} x\right\|+t^{2}\left\langle J_{k+1} v, v\right\rangle \\
= & t \sqrt{1-t^{2}} \sqrt{\frac{2}{k+2}} \sin \frac{\pi}{k+2}\left\|A^{k+1} x\right\|+t^{2} \cos \frac{\pi}{k+2} .
\end{aligned}
$$

To reach a contradiction, we need to find some $t_{0}, 0<t_{0}<1$, such that $\left\langle\left(J_{k+2} \otimes\right.\right.$ A) $\left.u_{t_{0}}, u_{t_{0}}\right\rangle>\cos (\pi /(k+2))$. This is the same as

$$
t_{0} \sqrt{1-t_{0}^{2}} \sqrt{\frac{2}{k+2}} \sin \frac{\pi}{k+2}\left\|A^{k+1} x\right\|>\left(1-t_{0}^{2}\right) \cos \frac{\pi}{k+2}
$$

or

$$
\frac{t_{0}}{\sqrt{1-t_{0}^{2}}}>\sqrt{\frac{k+2}{2}} \frac{\cot \frac{\pi}{k+2}}{\left\|A^{k+1} x\right\|} .
$$

Since $\lim _{t \rightarrow 1^{-}} t / \sqrt{1-t^{2}}=\infty$, the existence of such a $t_{0}$ is guaranteed. On the other hand, we also have

$$
\left\langle\left(J_{k+2} \otimes A\right) u_{t_{0}}, u_{t_{0}}\right\rangle \leq w\left(J_{k+2} \otimes A\right) \leq\left\|J_{k+2}\right\| w(A)=w(A)=\cos \frac{\pi}{k+2},
$$


hence a contradiction. Thus we must have $A^{k+1} x=0$. Let $K$ be the subspace of $\mathbb{C}^{n}$ generated by $x, A x, \ldots, A^{k} x$. Then $A K \subseteq K$. If $A^{\prime}$ is the restriction of $A$ to $K$, then $A^{\prime k+1}=0$ and $\left\|A^{\prime j} x\right\|=\left\|A^{j} x\right\|=1$ for all $j, 0 \leq j \leq k$. Hence $\left\|A^{\prime j}\right\|=1$ for all such $j$ 's. Together with $A^{\prime k+1}=0$, this says that $p_{A^{\prime}}=k$ and thus $\operatorname{dim} K=k+1$ by Proposition 1.2 (a). Therefore, $A^{\prime}$ is unitarily similar to a matrix of the form $\left[a_{i j}\right]_{i, j=1}^{k+1}$ with $a_{i j}=0$ for all $i \geq j$. Since $1=\left\|A^{\prime k}\right\|=\left|a_{12} \cdots a_{k, k+1}\right|$, we infer that $\left|a_{12}\right|=\cdots=\left|a_{k, k+1}\right|=1$, and thus all the other $a_{i j}$ 's are zero. Therefore, $\left[a_{i j}\right]_{i, j=1}^{k+1}$, and hence $A^{\prime}$, is unitarily similar to $J_{k+1}$. Then $A$ is unitarily similar to a matrix of the form

$$
\left[\begin{array}{c|ccc} 
& \multicolumn{3}{|c}{0} \\
J_{k+1} & \multicolumn{2}{|c}{0} \\
& b_{1} & \cdots & b_{n-k-1} \\
\hline & c_{1} & & * \\
0 & & \ddots & \\
& * & & c_{n-k-1}
\end{array}\right] .
$$

To show that all the $b_{j}$ 's are zero, we appeal to Lemma 2.11. Indeed, for each $j$, $1 \leq j \leq n-k-1$, consider the $(k+2)$-by- $(k+2)$ matrix

$$
A_{j}=\left[\begin{array}{c|c} 
& 0 \\
J_{k+1} & \vdots \\
& 0 \\
& b_{j} \\
\hline 0 & c_{j}
\end{array}\right] .
$$

If $b_{j} \neq 0$, then $w\left(A_{j}\right)>w\left(J_{k+1}\right)=\cos (\pi /(k+2))$ by Lemma 2.11, which contradicts $w\left(A_{j}\right) \leq w(A)=\cos (\pi /(k+2))$. This proves (a) $\Rightarrow(\mathrm{b})$.

Theorem 2.10 generalizes the classical result of Williams and Crimmins [17] for $k=1$. The following corollary is for $k=n-1$. Part of it has been proven in [19]: the equivalence of (b) and (c) is in [19, Theorem 1] and that of (b) and (d) in [19, p. $352]$. 
Corollary 2.12. The following conditions are equivalent for an n-by-n matrix A with $\|A\|=1$ :

(a) $\left\|A^{n-1}\right\|=1$ and $w(A)=\cos (\pi /(n+1))$,

(b) A is unitarily similar to $J_{n}$,

(c) $W(A)=\{z \in \mathbb{C}:|z| \leq \cos (\pi /(n+1))\}$,

(d) $\left\|A^{n-1}\right\|=1$ and $A^{n}=0_{n}$, and

(e) $p_{A}=n_{A}=n-1$.

Proof. The equivalence of (a) and (b) is by Theorem 2.10. The other implications are either in [19] or trivial.

Note that, in the preceding corollary, the conditions that $\|A\|=1$ and $w(A)=$ $\cos (\pi /(n+1))$ for an $n$-by- $n$ matrix $A$ are not sufficient to guarantee that $A$ be unitarily similar to $J_{n}$. One example is $A=J_{n-1} \oplus[\cos (\pi /(n+1))]$.

We end this section with a characterization of matrices $A$ satisfying $p_{A}=n_{A}$. This is related to the previous results.

Theorem 2.13. Let $A$ be an $n$-by-n matrix with $\|A\|=1$. Then

(a) A satisfies $p_{A}=n_{A}(\leq \infty)$ if and only if either it has a unitary part or is unitarily similar to a direct sum $J_{k+1} \oplus B$, where $k=p_{A}<\infty$ and $B^{k+1}=$ $0_{n-k-1}$, and

(b) if $p_{A}=n_{A}(\leq \infty)$, then $w(A \otimes A)=w(A)$ holds, but not conversely.

Proof. (a) For the necessity, we may assume, in view of Proposition 1.2 (c), that $k \equiv p_{A}=n_{A}<\infty$ and prove that $A$ is unitarily similar to the asserted direct sum. 
Since $A^{k+1}=0_{n}, A$ is unitarily similar to a block matrix $A^{\prime}$ of the form $\left[A_{i j}\right]_{i, j=1}^{k+1}$ with $A_{i j}=0$ for $1 \leq j \leq i \leq k+1$. Hence

$$
A^{\prime k}=\left[\begin{array}{cccc}
0 & \cdots & 0 & \prod_{i=1}^{k} A_{i, i+1} \\
& 0 & & 0 \\
& & \ddots & \vdots \\
& & & 0
\end{array}\right] .
$$

Since $\left\|A^{\prime k}\right\|=\left\|A^{k}\right\|=\|A\|^{k}=1$, we have $\left\|\prod_{i=1}^{k} A_{i, i+1}\right\|=1$. Let $x$ be a unit vector such that $\left\|\left(\prod_{i=1}^{k} A_{i, i+1}\right) x\right\|=1$. Then $\left\|\left(\prod_{i=j}^{k} A_{i, i+1}\right) x\right\|=1$ for all $j, 1 \leq j \leq k$. Let $\left\{e_{1}, \ldots, e_{k+1}\right\}$ be the standard basis for $\mathbb{C}^{k+1}$, and let $x_{j}=e_{j} \otimes\left(\prod_{i=j}^{k} A_{i, i+1}\right) x$ if $1 \leq j \leq k$, and $x_{k+1}=e_{k+1} \otimes x$. Then $x_{1}, \ldots, x_{k+1}$ are orthonormal vectors in $\mathbb{C}^{n}$, and $A^{\prime} x_{1}=0$ and $A^{\prime} x_{j}=x_{j-1}$ for $2 \leq j \leq k+1$. Thus if $K$ is the subspace generated by $x_{1}, \ldots, x_{k+1}$, then $\operatorname{dim} K=k+1, A^{\prime} K \subseteq K$, and the restriction of $A^{\prime}$ to $K$ is unitarily similar to $J_{k+1}$. We infer from $\left\|A^{\prime}\right\|=1$ and $A^{\prime *} x_{k+1}=0$ that $K$ reduces $A^{\prime}$, and thus $A^{\prime}$ is unitarily similar to $J_{k+1} \oplus B$ with $B^{k+1}=0$.

For the converse, if $A$ has a unitary part, then $p_{A}=n_{A}=\infty$ by Proposition 1.2 (c). On the other hand, if $A$ is unitarily similar to $J_{k+1} \oplus B$ with the asserted properties, then $A^{k+1}=0$ implies that $p_{A} \leq n_{A} \leq k$. But

$$
\left\|A^{k}\right\|=\left\|J_{k+1}^{k} \oplus B^{k}\right\|=\max \left\{\left\|J_{k+1}^{k}\right\|,\left\|B^{k}\right\|\right\}=1=\|A\|^{k}
$$

and $\left\|A^{k+1}\right\|=0<1=\|A\|^{k+1}$ together yield $p_{A}=n_{A}=k$.

(b) If $A$ has a unitary part, then $w(A \otimes A)=w(A)$ by Proposition 2.1. On the other hand, if $A$ is unitarily similar to $J_{k+1} \oplus B$ as in (a), then $A \otimes A$ is unitarily similar to $\left(J_{k+1} \otimes J_{k+1}\right) \oplus\left(J_{k+1} \otimes B\right) \oplus\left(B \otimes J_{k+1}\right) \oplus(B \otimes B)$. Note that $w\left(J_{k+1} \otimes J_{k+1}\right)=w\left(J_{k+1}\right)$ by Proposition 2.8, and

$$
w\left(J_{k+1} \otimes B\right)=w\left(B \otimes J_{k+1}\right) \leq\left\|J_{k+1}\right\| w(B)=w(B)
$$

by Proposition 1.1. Since $B^{k+1}=0$ and $\|B\| \leq 1$, [21, Lemma 3 (a)] implies that $B$ can be dilated to the direct sum of $\operatorname{rank}\left(I-B^{*} B\right)$ copies of $J_{m}$ for some $m \leq k+1$. 
Thus $w(B) \leq w\left(J_{m}\right) \leq w\left(J_{k+1}\right)$. Combined with (1), this yields $w\left(J_{k+1} \otimes B\right) \leq$ $w\left(J_{k+1}\right)$. Also,

$$
w(B \otimes B) \leq\|B\| w(B) \leq w(B) \leq w\left(J_{k+1}\right) .
$$

Therefore,

$$
\begin{aligned}
w(A \otimes B) & =\max \left\{w\left(J_{k+1} \otimes J_{k+1}\right), w\left(J_{k+1} \otimes B\right), w(B \otimes B)\right\} \\
& =w\left(J_{k+1}\right) \\
& =\max \left\{w\left(J_{k+1}\right), w(B)\right\} \\
& =w(A) .
\end{aligned}
$$

That $w(A \otimes A)=w(A)$ does not imply $p_{A}=n_{A}$ is seen by $A=J_{2} \oplus[a]$, where $0<|a| \leq 1 / 2$, in which case, $\|A\|=1$ and $w(A \otimes A)=w(A)=1 / 2$, but $p_{A}=1$ and $n_{A}=\infty$.

The final result of this section is conditions for a matrix $A$ with $p_{A}=n_{A}$ so that it be unitarily similar to a block-shift matrix

$$
A^{\prime}=\left[\begin{array}{cccc}
0 & A_{1} & & \\
& 0 & \ddots & \\
& & \ddots & A_{k} \\
& & & 0
\end{array}\right]
$$

with $\left\|A_{1} \cdots A_{k}\right\|=\|A\|$.

Proposition 2.14. Let $A$ be an n-by-n matrix with $p_{A}=n_{A} \equiv k<\infty$. If either (a) $k=1, n-2$ or $n-1$, or (b) $n=2,3,4$ or 5 , then $A$ is unitarily similar to the block-shift matrix $A^{\prime}$ in (2) with $\left\|A_{1} \cdots A_{k}\right\|=\|A\|$.

Proof. We may assume that $\|A\|=1$.

(a) If $k=n_{A}=1$, then $A^{2}=0_{n}$. Hence $A$ is unitarily similar to a block-shift matrix of the form $\left[\begin{array}{cc}0 & A_{1} \\ 0 & 0\end{array}\right]$ with $\left\|A_{1}\right\|=\|A\|$. 
If $k=p_{A}=n_{A}=n-1$ (resp., $n-2$ ), then Theorem 2.13 (a) implies that $A$ is unitarily similar to $J_{n}$ (resp., $J_{n-1} \oplus[0]$ ). The latter matrix plays the role of $A^{\prime}$ with $k=n-1$ (resp., $n-2)$ and $A_{1}=\cdots=A_{n-1}=[1]$ (resp., $A_{1}=\cdots=A_{n-3}=[1]$ and $\left.A_{n-2}=\left[\begin{array}{ll}1 & 0\end{array}\right]\right)$.

(b) In light of (a), we need only prove for $n=5$ and $k=2$. Invoking Theorem 2.13 to obtain the unitary similarity of $A$ and $J_{3} \oplus\left[\begin{array}{ll}0 & b \\ 0 & 0\end{array}\right]$, where $|b| \leq 1$. The latter matrix is permutationally similar to a block-shift matrix $A^{\prime}$ with $k=2, A_{1}=\left[\begin{array}{ll}1 & 0 \\ 0 & b\end{array}\right]$ and $A_{2}=\left[\begin{array}{l}1 \\ 0\end{array}\right]$. We obviously have $\left\|A_{1} A_{2}\right\|=\left\|\left[\begin{array}{l}1 \\ 0\end{array}\right]\right\|=1=\|A\|$.

We remark that the preceding proposition fails for $n=6$ and $k=2$. Here is an example. Let $A=J_{3} \oplus B$, where

$$
B=b\left[\begin{array}{lll}
0 & 1 & 1 \\
0 & 0 & 1 \\
0 & 0 & 0
\end{array}\right]
$$

with $b=\sqrt{2 /(3+\sqrt{5})}$. Then $\left\|A^{2}\right\|=1=\|A\|^{2}$ and $A^{3}=0_{6}$. This shows that $p_{A}=n_{A}=2$. Since $w(B)=2 b>\sqrt{2} / 2=w\left(J_{3}\right)$ and $w(B)$ is not a circular disc centered at the origin (cf. [13, Theorem $4.1(2)]$ ), we infer that nor is $W(A)$ (= the convex hull of $\left.W\left(J_{3}\right) \cup W(B)\right)$. This implies that $A$ cannot be unitarily similar to a block-shift matrix.

\section{Nonnegative Matrices}

Recall that a matrix $A=\left[a_{i j}\right]_{i, j=1}^{n}$ is nonnegative (resp., positive), denoted by $A \succcurlyeq 0$ (resp., $A \succ 0$ ), if $a_{i j} \geq 0$ (resp., $a_{i j}>0$ ) for all $i$ and $j$. Two $n$-by- $n$ matrices $A$ and $B$ are permutationally similar if there is an $n$-by- $n$ permutation matrix $P$ (one with 
each row and column has exactly one 1 and all other entries 0 ) such that $P^{T} A P=B$. $A$ is said to be (permutationally) reducible if either $A$ is the 1-by-1 zero matrix or $n \geq 2$ and it is permutationally similar to a matrix of the form $\left[\begin{array}{cc}B & C \\ 0 & D\end{array}\right]$, where $B$ and $D$ are square matrices; otherwise, it is (permutationally) irreducible. It is known that if $A$ is nonnegative with $\operatorname{Re} A$ irreducible, then it is permutationally similar to a block-shift matrix if and only if its numerical range is a circular disc centered at the origin (cf. [16, Theorem $1(\mathrm{a}) \Leftrightarrow(\mathrm{r})]$ ). Other properties of nonnegative matrices can be found in [11, Section 6.2 and Chapter 8].

The main result of this section is the following theorem, which essentially generalizes Theorem 2.5 .

Theorem 3.1. Let $A$ be an n-by-n matrix and $B$ an $m$-by-m nonnegative matrix with Re $B$ irreducible. Then the following conditions are equivalent:

(a) $w(A \otimes B)=\|A\| w(B)$,

(b) either $p_{A}=\infty$ or $n_{B} \leq p_{A}<\infty$ and $W(B)$ is a circular disc centered at the origin, and

(c) either $p_{A}=\infty$ or $n_{B} \leq p_{A}<\infty$ and $B$ is permutationally similar to a blockshift matrix

$$
\left[\begin{array}{cccc}
0 & B_{1} & & \\
& 0 & \ddots & \\
& & \ddots & B_{k} \\
& & & 0
\end{array}\right]
$$

with $k=n_{B}$.

For its proof, we need the following two lemmas.

Lemma 3.2. Let $A=\left[a_{i j}\right]_{i, j=1}^{n}$ be a nonnegative matrix. Then the following hold: 
(a) The index $n_{A}$ is finite if and only if there is no sequence of indices $i_{0}, i_{1}, \ldots, i_{k-1}, i_{k}(k \geq$ 1) with $i_{0}=i_{k}$ such that $a_{i_{0} i_{1}}, \ldots, a_{i_{k-1} i_{k}}$ are all nonzero. In particular, we have $n_{A}=\sup \left\{k \geq 1\right.$ : there are distinct $i_{j}, 0 \leq j \leq k$, such that $a_{i_{j} i_{j+1}} \neq$ 0 for all $j\}$.

(b) $n_{A}=\infty$ if and only if there is a $k \geq 1$ such that some diagonal entry of $A^{k}$ is nonzero.

(c) If $a_{i i} \neq 0$ for some $i, 1 \leq i \leq n$, then $n_{A}=\infty$.

(d) If $A$ is irreducible, then $n_{A}=\infty$.

(e) If $A$ is the block-shift matrix

$$
\left[\begin{array}{cccc}
0_{n_{1}} & A_{1} & & \\
& 0_{n_{2}} & \ddots & \\
& & \ddots & A_{k} \\
& & & 0_{n_{k+1}}
\end{array}\right] \text { on } \mathbb{C}^{n}=\mathbb{C}^{n_{1}} \oplus \cdots \oplus \mathbb{C}^{n_{k+1}}
$$

and $\operatorname{Re} A$ is irreducible, then $k=n_{A}$.

Proof. (a) Assume first that the indices $i_{0}, i_{1}, \ldots, i_{k-1}, i_{k}=i_{0}(k \geq 1)$ are such that $a_{i_{0} i_{1}}, \ldots, a_{i_{k-1} i_{k}} \neq 0$. [11, Theorem 6.2.16] says that this is the case if and only if $\left(A^{k}\right)_{i_{0} i_{0}}$, the $\left(i_{0}, i_{0}\right)$-entry of $A^{k}$, is nonzero. Hence $A^{k} \neq 0_{n}$. Similarly, considering the sequence $i_{0}, \ldots, i_{k}, i_{1}, \ldots, i_{k}, \ldots, i_{1}, \ldots, i_{k}$ of $\ell k+1$ indices for any $\ell \geq 1$, we also obtain $A^{\ell k} \neq 0_{n}$. It follows that $n_{A}=\infty$. Conversely, assume that $n_{A}=\infty$. Then $A^{k} \neq 0_{n}$ for some $k \geq n$. [11, Theorem 6.2.16] yields that, for some $i$ and $j$, there are indices $i_{0}=i, i_{1}, \ldots, i_{k-1}, i_{k}=j$ such that $a_{i_{0} i_{1}}, \ldots, a_{i_{k-1} i_{k}}$ are all nonzero. By the pigeonhole principle, we infer that $i_{s}=i_{t}$ for some $s$ and $t, 0 \leq s<t \leq k$. Then $i_{s}, \ldots, i_{t}$ are such that $i_{s}=i_{t}$ and $a_{i_{s} i_{s+1}}, \ldots, a_{i_{t-1} i_{t}} \neq 0$. This proves the converse. The expression for $n_{A}$ is an easy consequence of [11, Theorem 6.2.16] and the above arguments. So are (b) and (c). 
(d) Note that the irreducibility of $A$ is equivalent to the existence, for every distinct pair $i$ and $j$, of indices $i_{0}=i, i_{1}, \ldots, i_{k-1}, i_{k}=j(k \geq 1)$ such that $a_{i_{0} i_{1}}, \ldots, a_{i_{k-1} i_{k}}$ are all nonzero. Combining such indices from $i$ to $j$ with those from $j$ to $i$ yields one from $i$ to $i$ with the corresponding entries nonzero. Thus $n_{A}=\infty$ by 11, Theorem 6.2.16] and (b).

(e) Since $A^{k+1}=0_{n}$, we have $n_{A} \leq k$. If $n_{A}<k$, then $A^{k}=0_{n}$, which implies that $A_{1} \cdots A_{k}=0$. If there are any nonzero $a_{i_{0} i_{1}}, a_{i_{1} i_{2}}, \ldots, a_{i_{k-1} i_{k}}$, where $\left(\sum_{j=1}^{\ell} n_{j}\right)+1 \leq$ $i_{\ell} \leq \sum_{j=1}^{\ell+1} n_{j}$ for $0 \leq \ell \leq k$, then the $\left(i_{0}, n_{k+1}-\left(n-i_{k}\right)\right)$-entry of $A_{1} \cdots A_{k}$, being larger than or equal to $\prod_{j=0}^{k-1} a_{i_{j} i_{j+1}}$, is nonzero, which contradicts the zeroness of the product $A_{1} \cdots A_{k}$. Thus no such nonzero sequence exists. This results in the reducibility of $\operatorname{Re} A$, a contradiction. Hence we must have $n_{A}=k$.

We remark that the conditions in the preceding lemma can all be expressed equivalently in terms of the directed graph associated with the matrix $A$ (cf. [11, Section $6.2])$.

Lemma 3.3. Let $A$ and $B$ be $n$-by-n and $m$-by-m matrices, respectively. If $B$ is unitarily similar to a block-shift matrix

$$
\left[\begin{array}{cccc}
0_{m_{1}} & B_{1} & & \\
& 0_{m_{2}} & \ddots & \\
& & \ddots & B_{k} \\
& & & 0_{m_{k+1}}
\end{array}\right] \text { on } \mathbb{C}^{m}=\mathbb{C}^{m_{1}} \oplus \cdots \oplus \mathbb{C}^{m_{k+1}}
$$

with $k \leq p_{A} \leq \infty$, then $w(A \otimes B)=\|A\| w(B)$.

Proof. We may assume that $\|A\|=1$ and $B$ is equal to the block-shift matrix (3). Since $k \leq p_{A} \leq \infty$, we have $\left\|A^{k}\right\|=\|A\|^{k}=1$. Let $x$ be a unit vector in $\mathbb{C}^{n}$ such that $\left\|A^{k} x\right\|=1$, and let $y=\left[\begin{array}{lll}y_{1} & \ldots & y_{k+1}\end{array}\right]^{T}$, where $y_{j}$ is in $\mathbb{C}^{m_{j}}, 1 \leq j \leq k+1$, be a unit vector in $\mathbb{C}^{m}$ such that $|\langle B y, y\rangle|=w(B)$. Let $u=\left[\begin{array}{llll}y_{1} \otimes A^{k} x & y_{2} \otimes A^{k-1} x & \ldots & y_{k+1} \otimes x\end{array}\right]^{T}$. 
Then $u$ is a vector in $\mathbb{C}^{m} \otimes \mathbb{C}^{n}$ with

$$
\begin{aligned}
\|u\| & =\left(\sum_{j=1}^{k+1}\left\|y_{j} \otimes A^{k-j+1} x\right\|^{2}\right)^{1 / 2}=\left(\sum_{j=1}^{k+1}\left\|y_{j}\right\|^{2}\left\|A^{k-j+1} x\right\|^{2}\right)^{1 / 2} \\
& =\left(\sum_{j=1}^{k+1}\left\|y_{j}\right\|^{2}\right)^{1 / 2}=\|y\|=1 .
\end{aligned}
$$

Moreover, we have

$$
\begin{aligned}
& \begin{aligned}
& |\langle(B \otimes A) u, u\rangle| \\
= & \left\langle\left[\begin{array}{cccc}
0_{m_{1} n} & B_{1} \otimes A & & \\
& 0_{m_{2} n} & \ddots & \\
& & \ddots & B_{k} \otimes A \\
& & & 0_{m_{k+1} n}
\end{array}\right]\left[\begin{array}{c}
y_{1} \otimes A^{k} x \\
y_{2} \otimes A^{k-1} x \\
\vdots \\
y_{k+1} \otimes x
\end{array}\right],\left[\begin{array}{c}
y_{1} \otimes A^{k} x \\
y_{2} \otimes A^{k-1} x \\
\vdots \\
y_{k+1} \otimes x
\end{array}\right]\right\rangle \mid
\end{aligned} \\
& =\left|\sum_{j=1}^{k}\left\langle\left(B_{j} y_{j+1}\right) \otimes\left(A^{k-j+1} x\right), y_{j} \otimes\left(A^{k-j+1} x\right)\right\rangle\right| \\
& =\left|\sum_{j=1}^{k}\left\langle B_{j} y_{j+1}, y_{j}\right\rangle\left\|A^{k-j+1} x\right\|^{2}\right| \\
& =\left|\sum_{j=1}^{k}\left\langle B_{j} y_{j+1}, y_{j}\right\rangle\right| \\
& =|\langle B y, y\rangle|=w(B) \text {. }
\end{aligned}
$$

This shows that $w(B) \leq w(B \otimes A)=w(A \otimes B)$. But $w(A \otimes B) \leq\|A\| w(B)=w(B)$ always holds by Proposition 1.1. Hence $w(A \otimes B)=w(B)$ as asserted.

We are now ready to prove Theorem 3.1 .

Proof of Theorem 3.1. For (a) $\Rightarrow(\mathrm{b})$, We assume that $\|A\|=1$ and $A$ is c.n.u. In view of Theorem 2.2 and Proposition 1.2 (c), we need only check that $w(A \otimes B)=w(B)$ implies $n_{B} \leq p_{A}(<\infty)$. Let $B=\left[b_{i j}\right]_{i, j=1}^{m}$, and let $x$ be a unit vector in $\mathbb{C}^{m} \otimes \mathbb{C}^{n}$ such that $w(B \otimes A)=|\langle(B \otimes A) x, x\rangle|$. If $x=\left[\begin{array}{lll}x_{1} & \ldots & x_{m}\end{array}\right]^{T}$, where $x_{j}$ is in $\mathbb{C}^{n}$ for 
$1 \leq j \leq m$, then

$$
\begin{aligned}
w(B) & =w(B \otimes A)=\left|\left\langle\left[b_{i j} A\right] x, x\right\rangle\right| \\
& \leq \sum_{i, j} b_{i j}\left|\left\langle A x_{j}, x_{i}\right\rangle\right| \\
& \leq \sum_{i, j} b_{i j}\left\|A x_{j}\right\|\left\|x_{i}\right\| \\
& \leq\|A\| \sum_{i, j} b_{i j}\left\|x_{j}\right\|\left\|x_{i}\right\| \\
& \leq\left\langle B x^{\prime}, x^{\prime}\right\rangle \\
& \leq w(B),
\end{aligned}
$$

where $x^{\prime}=\left[\left\|x_{1}\right\| \ldots\left\|x_{m}\right\|\right]^{T}$ is a unit vector in $\mathbb{C}^{m}$. This shows that the above inequalities are equalities throughout. Since $B \succcurlyeq 0$ and $\operatorname{Re} B$ is irreducible, there is a unique unit vector $y$ in $\mathbb{C}^{m}$ with $y \succ 0$ such that $\langle B y, y\rangle=w(B)$ (cf. [14, Proposition 3.3]). The equality in (6) yields that $x^{\prime}=y$ and thus $x_{j} \neq 0$ for all $j$. Also, the equalities in (41) and (5) imply that $\left|\left\langle A x_{j}, x_{i}\right\rangle\right|=\left\|A x_{j}\right\|\left\|x_{i}\right\|=\left\|x_{j}\right\|\left\|x_{i}\right\|$ for all those $b_{i j}$ 's with $b_{i j}>0$. Thus $A x_{j}=\lambda_{i j} x_{i}$ for some $\lambda_{i j}$ satisfying $\left|\lambda_{i j}\right|=\left\|x_{j}\right\| /\left\|x_{i}\right\|$. Assume first that $k \equiv n_{B}<\infty$. Thus $B^{k} \neq 0_{m}$. By Lemma 3.2 (a), there are distinct indices $i_{0}, \ldots, i_{k}$ such that $b_{i_{0} i_{1}}, \ldots, b_{i_{k-1} i_{k}}>0$. It thus follows from above that $A x_{i_{j}}=\lambda_{i_{j-1} i_{j}} x_{i_{j-1}}$ for $1 \leq j \leq k$. Hence $A^{k} x_{i_{k}}=\left(\prod_{j=1}^{k} \lambda_{i_{j-1} i_{j}}\right) x_{i_{0}}$. Since

$$
\left\|A^{k} x_{i_{k}}\right\|=\left(\prod_{j=1}^{k} \frac{\left\|x_{i_{j}}\right\|}{\left\|x_{i_{j-1}}\right\|}\right)\left\|x_{i_{0}}\right\|=\left\|x_{i_{k}}\right\|,
$$

we obtain $\left\|A^{k}\right\|=1$ or $p_{A} \geq k=n_{B}$. On the other hand, if $n_{B}=\infty$, then the same arguments as above with $k$ arbitrarily large yield that $p_{A}=\infty$, which contradicts our assumption that $A$ is c.n.u. This proves $(\mathrm{a}) \Rightarrow(\mathrm{b})$.

That $(\mathrm{b}) \Leftrightarrow(\mathrm{c})$ is a consequence of [16, Theorem $1(\mathrm{a}) \Leftrightarrow(\mathrm{r})]$, and $(\mathrm{c}) \Rightarrow(\mathrm{a})$ is by Lemma 3.2 (e) and Lemma 3.3.

Note that, in Theorem 3.1, the implication (a) $\Rightarrow(\mathrm{b})$ or $(\mathrm{a}) \Rightarrow(\mathrm{c})$ is no longer true if $B$ is nonnegative but without the irreducibility of $\operatorname{Re} B$. One example is 
$A=B=J_{2} \oplus[a]$, where $0<a \leq 1 / 2$ (cf. the end of the proof of Theorem 2.13 (b)). The next example shows that the same can be said if $B$ is not nonnegative but $\operatorname{Re} B$ is irreducible.

Example 3.4. Let $A=J_{3}$ and

$$
B=\left[\begin{array}{ccc}
0 & -\sqrt{2} & 1 \\
0 & 0 & 1 \\
0 & 0 & \sqrt{2} / 2
\end{array}\right] .
$$

Then $\operatorname{Re} B$ is easily seen to be irreducible. We now show that $W(B)=\overline{\mathbb{D}}$. This is seen via [13, Corollary 2.5] by letting $u=0$ and $\lambda=\sqrt{2} / 2$ therein and checking that

$$
\operatorname{tr}\left(B^{*} B^{2}\right)=\operatorname{tr}\left[\begin{array}{ccc}
0 & 0 & 0 \\
0 & 0 & 1 \\
0 & 0 & \sqrt{2} / 4
\end{array}\right]=\frac{\sqrt{2}}{4}=\lambda|\lambda|^{2}
$$

and $\operatorname{tr}\left(B^{*} B\right)=9 / 2 \geq 5|\lambda|^{2}$, where $\operatorname{tr}(\cdot)$ denotes the trace of a matrix. We next prove that 1 is an eigenvalue of $\operatorname{Re}(A \otimes B)$. Indeed, since

$$
\operatorname{Re}(A \otimes B)=\frac{1}{2}\left[\begin{array}{ccc}
0_{3} & B & 0_{3} \\
B^{*} & 0_{3} & B \\
0_{3} & B^{*} & 0_{3}
\end{array}\right],
$$

we need to check that

$$
\operatorname{det}\left[\begin{array}{ccc}
2 I_{3} & -B & 0_{3} \\
-B^{*} & 2 I_{3} & -B \\
0_{3} & -B^{*} & 2 I_{3}
\end{array}\right]=0
$$

By a repeated use of the Schur decomposition, the above determinant is seen to be 
equal to

$$
\begin{aligned}
& \operatorname{det}\left(2 I_{3}\right) \operatorname{det}\left(\left[\begin{array}{cc}
2 I_{3} & -B \\
-B^{*} & 2 I_{3}
\end{array}\right]-\left[\begin{array}{c}
-B^{*} \\
0_{3}
\end{array}\right]\left(\frac{1}{2} I_{3}\right)\left[-B \quad 0_{3}\right]\right) \\
= & 8 \operatorname{det}\left[\begin{array}{cc}
2 I_{3}-(1 / 2) B^{*} B & -B \\
-B^{*} & 2 I_{3}
\end{array}\right] \\
= & 8 \operatorname{det}\left(4 I_{3}-B^{*} B-B B^{*}\right) \\
= & 8 \operatorname{det}\left[\begin{array}{ccc}
1 & -1 & -\sqrt{2} / 2 \\
-1 & 1 & \sqrt{2} / 2 \\
-\sqrt{2} / 2 & \sqrt{2} / 2 & 1
\end{array}\right] \\
= & 0
\end{aligned}
$$

as required. Since $W(A \otimes B)$ is a circular disc centered at the origin (by the unitary similarity of $A \otimes B$ and $e^{i \theta}(A \otimes B)$ for all real $\left.\theta\right)$ and $w(A \otimes B) \leq\|A\| w(B)=1$, we infer from $1 \in \sigma(\operatorname{Re}(A \otimes B))$ that $W(A \otimes B)=\overline{\mathbb{D}}$. Hence $w(A \otimes B)=1=\|A\| w(B)$. But, obviously, we have $n_{B}=\infty$ and $p_{A}=2$.

The next corollary gives a more concrete equivalent condition, in terms of blockshift matrices, for $w(A \otimes B)=\|A\| w(B)$ when $A=B \succcurlyeq 0$ and $\operatorname{Re} B$ is irreducible.

Corollary 3.5. Let $A$ be an n-by-n nonnegative matrix with $\operatorname{Re} A$ irreducible. Then the following conditions are equivalent:

(a) $w(A \otimes A)=\|A\| w(A)$,

(b) $p_{A}=n_{A}(\leq \infty)$, and

(c) either $A$ is unitarily similar to $[a] \oplus A^{\prime}$ with $|a| \geq\left\|A^{\prime}\right\|$, or $A$ is permutationally similar to a block-shift matrix

$$
A^{\prime \prime}=\left[\begin{array}{cccc}
0 & A_{1} & & \\
& 0 & \ddots & \\
& & \ddots & A_{k} \\
& & & 0
\end{array}\right]
$$


with $\left\|A_{1} \cdots A_{k}\right\|=\|A\|$.

Proof. We may assume that $\|A\|=1$. The implication (a) $\Rightarrow(\mathrm{b})$ is by Theorem 3.1 and Proposition 1.3 (d). For (b) $\Rightarrow$ (c), if $p_{A}=n_{A}=\infty$, then $A$ has a unitary part by Proposition 1.2 (c), and hence $A$ is unitarily similar to $[a] \oplus A^{\prime}$ with $|a|=1 \geq\left\|A^{\prime}\right\|$ as asserted. On the other hand, if $p_{A}=n_{A}<\infty$, then $w(A \otimes A)=w(A)$ by Theorem 2.13 (b). Hence Theorem 2.2 implies that $W(A)$ is a circular disc centered at the origin. For a nonnegative $A$ with $\operatorname{Re} A$ irreducible, this is equivalent to $A$ being permutationally similar to the block-shift matrix $A^{\prime \prime}$ (cf. [16, Theorem $\left.1(\mathrm{a}) \Leftrightarrow(\mathrm{r})\right]$ ). As $n_{A^{\prime \prime}}=k$ by Lemma 3.2 (e), we also have $p_{A}=k$. Thus $\left\|A^{k}\right\|=\|A\|^{k}=1$, which yields that $\left\|A_{1} \cdots A_{k}\right\|=1=\|A\|$ as required. Finally, for (c) $\Rightarrow$ (a), if $A$ is unitarily similar to $[a] \oplus A^{\prime}$ with $|a| \geq\left\|A^{\prime}\right\|$, then $w(A \otimes A)=w(A)$ by Lemma 2.1 . On the other hand, if $A$ is permutationally similar to the block-shift matrix $A^{\prime \prime}$ with $\left\|A_{1} \cdots A_{k}\right\|=1$, then

$$
\left\|A^{k}\right\|=\left\|A^{\prime \prime k}\right\|=\left\|A_{1} \cdots A_{k}\right\|=1=\|A\|^{k} .
$$

Thus $p_{A} \geq k=n_{A}$. The equality $w(A \otimes A)=w(A)$ then follows from Theorem 3.1.

Corollary 3.6. Let $A=\left[a_{i j}\right]_{i, j=1}^{n}$, where $a_{i j} \geq 0$ for all $i$ and $j, a_{i j}=0$ for $i \geq j$, and $a_{i, i+1}>0$ for all $i$. Then the following conditions are equivalent:

(a) $w(A \otimes A)=\|A\| w(A)$,

(b) $p_{A}=n_{A}=n-1$, and

(c) $a_{12}=\cdots=a_{n-1, n}$ and $a_{i j}=0$ for all other pairs of $i$ and $j$.

Proof. In this case, $A$ is nonnegative, $\operatorname{Re} A$ is irreducible and $n_{A}=n-1$. Consequently, Corollary 3.5 yields the equivalence of (a), (b) and the condition (c') that $A$ is permutationally similar to a block-shift matrix $A^{\prime \prime}$ as in Corollary 3.5 
(c). Since $k=n_{A^{\prime \prime}}=n_{A}$ by Lemma 3.2 (e), $A^{\prime \prime}$ is necessarily equal to $A$ with $\left|a_{12} \cdots a_{n-1, n}\right|=\|A\|$ and $a_{i j}=0$ for all other pairs of $i$ and $j$. The norm condition above yields that $a_{12}=\cdots=a_{n-1, n}=\|A\|$. Thus ( $\mathrm{c}^{\prime}$ ) is the same as (c), and we have the equivalence of (a), (b) and (c).

\section{Acknowledgements}

This research was partially supported by the National Science Council of the Republic of China under projects NSC-101-2115-M-008-006, NSC-101-2115-M-009-001 and NSC-101-2115-M-009-004 of the respective authors. P. Y. Wu was also supported by the MOE-ATU. This paper was presented by him at the 4th International Conference on Matrix Analysis and Applications in Konya, Turkey. He thanks the organizers for their works with the conference. 


\section{References}

[1] H.-L. Gau, Numerical ranges of reducible companion matrices, Linear Algebra Appl. 432 (2010), pp. 1310-1321.

[2] H.-L. Gau and P. Y. Wu, Numerical range of $S(\phi)$, Linear Multilinear Algebra 45 (1998), pp. 49-73.

[3] H.-L. Gau and P. Y. Wu, Condition for the numerical range to contain an elliptic disc, Linear Algebra Appl. 364 (2003), pp. 213-222.

[4] H.-L. Gau and P. Y. Wu, Finite Blaschke products of contractions, Linear Algebra Appl. 368 (2003), pp. 359-370.

[5] H.-L. Gau and P. Y. Wu, Companion matrices: reducibility, numerical ranges and similarity to contractions, Linear Algebra Appl. 383 (2004), pp. 127-142.

[6] H.-L. Gau and P. Y. Wu, Numerical ranges of companion matrices, Linear Algebra Appl. 421 (2007), pp. 202-218.

[7] K. Gustafson and D. K. M. Rao, Numerical Range. The Field of Values of Linear Operators and Matrices, Springer, New York, 1997.

[8] U. Haagerup and P. de la Harpe, The numerical radius of a nilpotent operator on a Hilbert space, Proc. Amer. Math. Soc. 115 (1992), pp. 371-379.

[9] P. R. Halmos, A Hilbert Space Problem Book, 2nd ed., Springer, New York, 1982.

[10] J. A. R. Holbrook, Multiplicative properties of the numerical radius in operator theory, J. Reine Angew. Math. 237 (1969), pp. 166-174.

[11] R. A. Horn and C. R. Johnson, Matrix Analysis, Cambridge University Press, Cambridge, 1985.

[12] R. A. Horn and C. R. Johnson, Topics in Matrix Analysis, Cambridge University Press, Cambridge, 1991. 
[13] D. S. Keeler, L. Rodman and I. M. Spitkovsky, The numerical range of $3 \times 3$ matrices, Linear Algebra Appl. 252 (1997), pp. 115-139.

[14] C.-K. Li, B.-S. Tam and P. Y. Wu, The numerical range of a nonnegative matrix, Linear Algebra Appl. 350 (2002), pp. 1-23.

[15] V. Pták, Lyapunov equations and Gram matrices, Linear Algebra Appl. 49 (1983), pp. 33-55.

[16] B.-S. Tam and S. Yang, On matrices whose numerical ranges have circular or weak circular symmetry, Linear Algebra Appl. 302/303 (1999), pp. 193-221.

[17] J. P. Williams and T. Crimmins, On the numerical radius of a linear operator, Amer. Math. Monthly 74 (1967), pp. 832-833.

[18] P. Y. Wu, Unitary dilations and numerical ranges, J. Operator Theory 38 (1997), pp. $25-42$.

[19] P. Y. Wu, A numerical range characterization of Jordan blocks, Linear Multilinear Algebra 43 (1998), pp. 351-361.

[20] P. Y. Wu, Numerical ranges as circular discs, Applied Math. Lett. 24 (2011), pp. $2115-2117$.

[21] P. Y. Wu, H.-L. Gau and M.-C. Tsai, Numerical radius inequality for $C_{0}$ contractions, Linear Algebra Appl. 430 (2009), pp. 1509-1516. 